\title{
SOLVATION SIMPLIFIED
}

\section{Omar A. EI Seoud}

Instituto de Química, Universidade de São Paulo, CP 26077, 05513-970 São Paulo - SP, Brasil

Recebido em 24/5/10; aceito em 28/9/10; publicado na web em 16/11/10

\begin{abstract}
The effects of solvents on chemical phenomena is complex because there are various solute-solvent interaction mechanisms. Solvatochromism refers to the effects of solvents on the spectra of probes. The study of this phenomenon sheds light on the relative importance of the solvation mechanisms. Solvation in pure solvents is quantitatively analyzed in terms of a multi-parameter equation. In binary solvent mixtures, solvation is analyzed by considering the organic solvent, $\mathrm{S}$, water, $\mathrm{W}$, and a 1:1 hydrogen bonded species (S-W). The applications of solvatochromism to understand distinct chemical phenomena, reactivity and swelling of cellulose, is briefly discussed.
\end{abstract}

Keywords: solvation ; solvatochromism; solute-solvent interactions.

The need for understanding solvation is clear: most reactions are carried out in the liquid phase; the solvent is not a "spectator". It acts as a heat- and mass-transfer agent; it participates in proton transfers (for acid/base catalyzed reactions) and in the solvation of ions, dipolar species, etc. Consider the decomposition of 6-nitro-3carboxybenzisoxazole, whose reaction scheme is depicted in Figure 1. This example is particularly illustrative of the effects of solvents on reactivity because it is a simple, spontaneous decomposition reaction. Therefore, effects of changing the solvent on the observed rate constant, $\mathrm{k}_{\mathrm{obs}}$, can be unequivocally attributed to differences in solvation between the reactant state- where the negative charge is concentrated on the carboxylate anion- and the transition state, where the charge is dispersed over several atoms. The half-lives of this reaction in hexamethylphosphotriamide, acetonitrile, and water, are $0.001 \mathrm{~s}, 11.6 \mathrm{~min}$, and one day, respectively! ${ }^{1}$<smiles>O=C(O)c1noc2cc([N+](=O)[O-])ccc12</smiles>

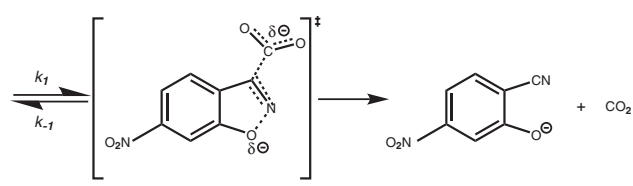

Figure 1. Schematic representation of the spontaneous decomposition of 6-Nitro-3-carboxybenzisoxazole. Reproduced from ref. 1 with permission of ACS

Can these large differences in $\mathrm{k}_{\text {obs }}$ be correlated with solvent properties? The results of such attempt is shown in Table 1, where the subscript (S) refers to solvent, $\varepsilon_{\mathrm{r}}, \mathrm{E}_{\mathrm{T}}(30), \alpha_{\mathrm{S}}, \beta_{\mathrm{S}}$ refer to the solvent relative permittivity, its empirical polarity, hydrogen-bond donation capacity or "acidity", and hydrogen-bond acceptance capacity or "basicity", respectively (vide infra for more discussion of the last three solvent descriptors).

Table 1 reveals that there is no correlation between $\log \mathrm{k}_{\mathrm{obs}}$ and any single solvent property. Inclusion of a second descriptor leads to a noticeable improvement in the regression analysis; a four-descriptor equation gives the best correlation coefficient. The conclusion from Table 1 is obvious: solvent effects on chemical reactivity, and presumably other phenomena, e.g., chemical equilibria and spectroscopic data, are complex; any successful correlation with a single solvent descriptor is, most certainly, fortuitous.

The following questions now arise: (i) What is the reason for this complex dependence of chemical phenomena on solvent properties?

*e-mail: elseoud@iq.usp.br

\#This paper is dedicated to Prof. Hans Viertler
Table 1. Correlations between $\log$ (observed rate constants, $\mathrm{k}_{\mathrm{obs}}$ ) for the spontaneous decomposition of 6-Nitro-3-carboxybenzisoxazole in different solvents with their properties., ${ }^{\mathbf{a}, \mathbf{b}}$

\begin{tabular}{lc}
\hline Solvent property & $\begin{array}{c}\text { Coefficients of the correlations between } \\
\log \mathrm{k}_{\mathrm{obs}} \text { and solvent property }\end{array}$ \\
\hline$f \varepsilon_{\mathrm{r}}=2\left(\varepsilon_{\mathrm{r}}-1\right) /\left(2 \varepsilon_{\mathrm{r}}+1\right)$ & $\mathbf{0 . 0 7 7 8}$ \\
$\mathrm{E}_{\mathrm{T}}(30)$ & 0.1572 \\
$\mathrm{E}_{\mathrm{T}}(30)+f \varepsilon_{\mathrm{r}}$ & 0.7864 \\
$\mathrm{E}_{\mathrm{T}}(30)+\alpha_{\mathrm{S}}$ & 0.7791 \\
$\mathrm{E}_{\mathrm{T}}(30)+\beta_{\mathrm{S}}$ & 0.5167 \\
$\mathrm{E}_{\mathrm{T}}(30)+\alpha_{\mathrm{S}}+\beta_{\mathrm{S}}$ & 0.8928 \\
$\mathrm{E}_{\mathrm{T}}(30)+f \varepsilon_{\mathrm{r}}+\alpha_{\mathrm{S}}$ & 0.8916 \\
$\mathrm{E}_{\mathrm{T}}(30)+f \varepsilon_{\mathrm{r}}+\alpha_{\mathrm{S}+} \beta_{\mathrm{S}}$ & $\mathbf{0 . 9 4 8 5}$ \\
\hline
\end{tabular}

a- Values of $\mathrm{k}_{\mathrm{obs}}$ were taken from ref. 1 ; $\mathrm{b}$ - the solvent properties include relative permittivity, $\varepsilon_{\mathrm{r}}$; empirical polarity, $\mathrm{E}_{\mathbf{T}}(30)$; acidity, $\alpha_{\mathrm{S}}$; and basicity, $\beta_{\mathrm{S}}$; $\mathrm{c}$ - the correlation coefficients are $(\mathrm{r})$ and $\left(\mathrm{r}^{2}\right)$ for linear, and multiple regression analysis, respectively.

(ii) Can the relative importance of each solute-solvent interaction be identified and quantified? (iii) How can we treat solvation in solvent mixtures? The objective of the following discussion is to offer a simplified, non-mathematical answer to the above-formulated questions. It is intended to help those who are interested in exploiting solvent effects without having to read lengthy, specialized literature. More discussion on the subject can be found in specialized books, and review articles. ${ }^{2-11}$

The reason for the multi-parameter dependence of chemical phenomena on solvent properties, point (i) above, is that several solutesolvent interactions come into play; their effects should be taken into account. These include both specific and non-specific interactions, e.g., hydrogen-bonding, dipolar-interactions (ion-dipole, dipoledipole, dipole-induced dipole), and dispersion or London interactions.

With regard to (ii), there have been several approaches to quantify the dependence of chemical phenomena on solvent properties, most notably the Taft-Kamlet-Abboud Equation: ${ }^{12}$

$\mathrm{SDP}=$ Constant $+\mathrm{a} \alpha_{\mathrm{S}}+\mathrm{b} \beta_{\mathrm{S}}+\mathrm{s}\left(\pi^{*}{ }_{\mathrm{S}}+\mathrm{d} \delta\right)+\mathrm{h}\left(\delta_{\mathrm{H}}^{2}\right)$

Here a solvent dependent phenomenon, SDP, such as rate cons- 
tant, equilibrium constant, spectroscopic shift, is modeled as a linear combination of two hydrogen-bonding terms, in which the solvent acts as a hydrogen-bond donor ( $\mathrm{a} \alpha_{\mathrm{S}}$ ), or hydrogen-bond acceptor (b $\beta_{\mathrm{s}}$ ), a dipolarity/polarizability term $\left[\mathrm{s}\left(\pi_{\mathrm{s}} \mathrm{s}+\mathrm{d} \delta\right)\right]$, and a cavity term $\left(\mathrm{h}\left(\delta_{\mathrm{H}}^{2}\right)\right.$, related to Hildebrand solubility parameter. The term (d $\delta$ ) is a correction for $\pi^{*}$ s. A procedure has been given in order to separate solvent dipolarity from its polarizability. ${ }^{13}$ The parameters $\alpha_{\mathrm{s}}, \beta_{\mathrm{s}}$, and $\pi_{\mathrm{s}}^{*}$ are known as solvatochromic parameters because they are determined by using solvatochromic probes (vide infra). The latter are substances whose spectra, absorption or emission, are particularly sensitive to specific solvent properties (acidity, basicity, etc). The information about solvation is derived as follows: From the spectra of the probe in a series of solvents, an empirical solvent polarity scale, $\mathrm{E}_{\mathrm{T}}$ (probe) is calculated from Equation $2: 5$

$\mathrm{E}_{\mathrm{T}}($ probe $), \mathrm{kcal} / \mathrm{mol}=28591.5 / \lambda_{\max }(\mathrm{nm})$

This equation converts the electronic transition within the probe into the corresponding intra-molecular charge-transfer energy. These probes obey the Frank-Condon principle; hence the last term of Equation $1\left(\mathrm{~h}\left(\delta_{\mathrm{H}}^{2}\right)\right)$ is redundant. Values of $\mathrm{E}_{\mathrm{T}}$ (probe) are then rationalized in terms of non-specific and specific probe-solvent interaction mechanisms, vide supra; e.g.: ${ }^{14}$

$\mathrm{E}_{\mathrm{T}}($ probe $)=$ Constant $+\mathrm{a} \alpha_{\mathrm{s}}+\mathrm{b} \beta_{\mathrm{s}}+\mathrm{s}\left(\pi^{*}{ }_{\mathrm{s}}+\mathrm{d} \delta\right)+\mathrm{p} \log \mathrm{P}_{\mathrm{S}}$

Where a new term $\left(\mathrm{p} \log \mathrm{P}_{\mathrm{S}}\right)$ has been introduced because the spectroscopic response of merocyanine probes is sensitive to solvent lipophilicity, as measured by the empirical scale $\log \mathrm{P}_{\mathrm{S}}$ (= partition coefficient of a substance between (mutually saturated) 1-octanol and water: $\left.\log \mathrm{P}=\log \left([\text { substance }]_{1-\text { octanol }} /[\text { substance }]_{\text {water }}\right)\right)$. Figure 2 shows some solvatochromic probes that the author's group has employed in order to study solvents and their mixtures, along with their $\mathrm{pKa}$ in water and $\log \mathrm{P}$.

The molecular structures of these probes merits a comment, in order to demonstrate how their judicious choice can be exploited in order to extract information about the relative importance of solute-solvent interactions. Whereas the $\mathrm{pKa}$ of merocyanines 2 to 5 $\left(\mathrm{MePMeBr}_{2}\right.$ to $\left.\mathrm{OcPMeBr}_{2}\right)$ is the same, their $\log \mathrm{P}$ vary by 2.86 units (relative to water, $\mathrm{OcPMeBr}_{2}$ is 724 times more soluble in 1-octanol than $\mathrm{MePMeBr}_{2}$ ). This difference has been employed for evaluation of the relative importance of solute-solvent solvophobic interactions, for binary mixtures of water with both molecular solvents, ${ }^{14}$ and ionic liquids, ILs. ${ }^{15}$ Although RB is 3467 time more basic than WB, the susceptibility of their solvation toward solvent acidity is almost the same. The reason is that the regression coefficient (a) of Equation 3 for RB is attenuated because the site of hydrogen bonding (the oxygen atom of the phenolate ion) is sterically hindered, due to the two ortho phenyl rings. Indeed, H-bonding ability of RB depends on the acidity and steric hindrance of the proton donor, e.g., it does not form H-bond to 2,6-di-tert-butyl-4-methylphenol. ${ }^{16}$ For $\mathrm{WB}$, this severe crowding around the phenolate oxygen is absent. Additionally, its $\mathrm{C}-\mathrm{Cl}$ bonds are appreciably ionic, so that the chlorine atoms may form additional hydrogen bonds with the solvent, ${ }^{17}$ akin to 2,6-dichlorophenol. ${ }^{18}$ Figure 3 shows the DFT-optimized geometries of some of the probes depicted in Figure 2.

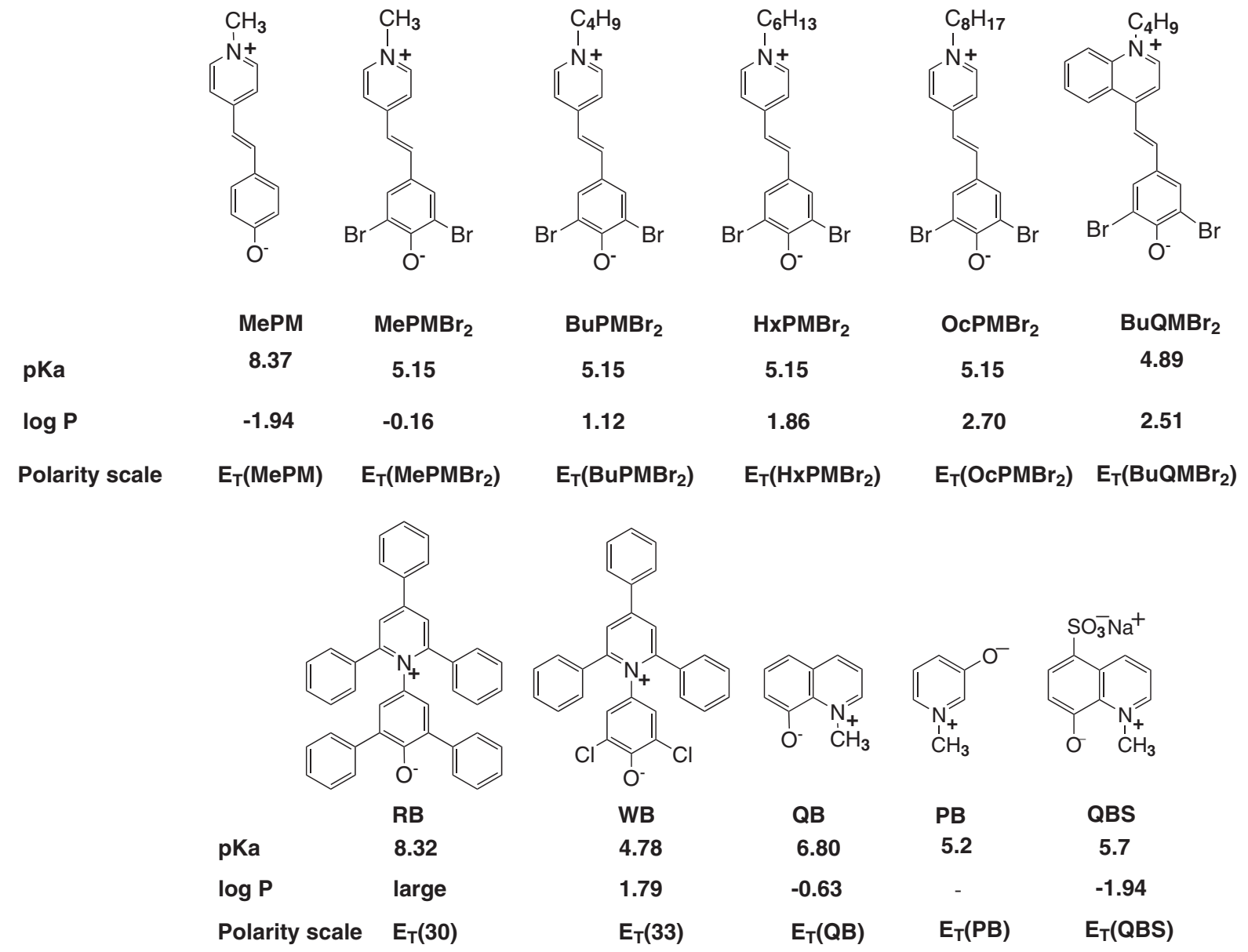

Figure 2. Molecular structures of zwitterionic solvatochromic indicators, along with the pKa of their conjugate acids in water; and log P. The acronyms of the probes and the corresponding empirical polarity scales are also indicated. Reproduced from ref. 10 with permission of IUPAC 


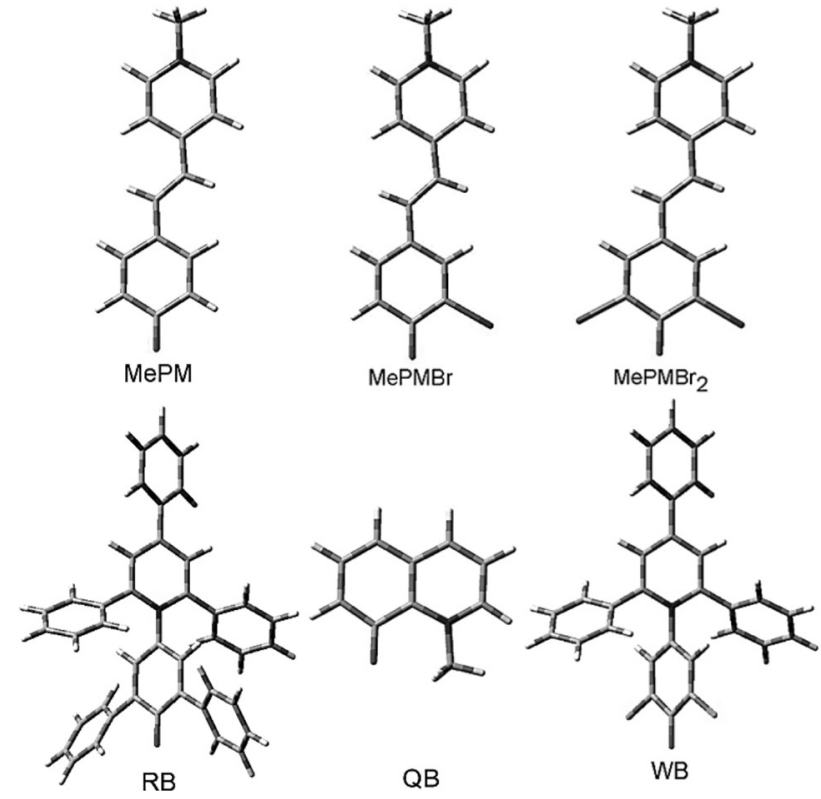

Figure 3. DFT-optimized geometries of some of the probes studied. Reproduced from ref. 17 with permission of ACS

The pair QB and QBS is interesting because they have the same intra-molecular charge transfer $\left(\mathrm{E}_{\mathrm{T}}(\mathrm{QB}) \approx \mathrm{E}_{\mathrm{T}}(\mathrm{QBS})\right)$, but the former is zwitterionic, whereas the latter is anionic. That is, the difference of their responses to the properties of the medium can be unequivocally attributed to the presence of the negative charge (in QBS). This difference has been exploited in order to probe the interfacial region of cationic micelles $\left(\mathrm{RN}^{+}\left(\mathrm{CH}_{3}\right)_{3} \mathrm{X}^{*} ; \mathrm{X}^{-}=\mathrm{Br}^{-}\right.$or $\left.\mathrm{Cl}^{-}\right)$. The reason is that their solubilization sites in the micellar pseudo-phase is different, QBS exchanges with the surfactant counter-ion $\left(\mathrm{Br}^{-}\right.$or $\left.\mathrm{Cl}^{-}\right)$, therefore it samples the outer layer of the interfacial region. ${ }^{19}$

As an example of the determination of the relative importance of solute-solvent interaction mechanisms, consider the solvation of solvatochromic probes. The procedure in order to extract this information is as follows: ${ }^{10,11}$ we determine the effects of a group of solvents on the Uv-vis spectra of one or more probes; we apply Equation 2 in order to calculate $\mathrm{E}_{\mathrm{T}}$ (probe); we use Equation 3 in order to calculate the regression coefficients (a), (b), (s), and (p). Finally, we draw conclusions about the relative importance of solvent acidity, basicity, etc., from these regression coefficients, after their values are statistically standardized (i.e., from $\beta_{\text {statistical }}$ (regression coefficient)), in order to make them comparable. Values of $\alpha_{\mathrm{s}}, \beta_{\mathrm{s}}$, etc. are known for a myriad of solvents. Where not available, they can be determined by using appropriate solvatochromic indicators. Examples of the latter are 4-nitroanisol and RB (for determination of $\alpha_{\mathrm{s}}$ ); 4-nitroaniline and $N, N$-dimethyl-4-nitroaniline (for determination of $\beta_{\mathrm{s}}$ ), and $N, N$-dimethyl-4-nitroaniline (for determination of $\left.\pi{ }_{\mathrm{S}}\right){ }^{20}$ Table 2 shows some typical values. Whereas the solvation of the first three probes depends on solvent acidity, dipolarity/polarizability, and lipophilicity, that of the other three can be conveniently described in terms of solvent acidity and dipolarity/polarizability. The relative importance of $\alpha_{s}$ is understandable because of the formation of hydrogen-bonds between the solvent and the probe phenolate oxygen, as shown by ${ }^{1} \mathrm{H}$ NMR. ${ }^{21}$ The dependence on $\pi^{*}$ s is also expected because these probe have large dipole moments. In fact the (calculated) dipole moments, $\mu_{\text {(probe) }}$, for six of the probes shown in Figure 1 (RB, WB, QB, MePM; MePMBr, and MePMBr ${ }_{2}$ ) were found to correlate linearly with the regression coefficient $(\mathrm{s})$ of Equation 3, according to: ${ }^{17}$ $\mu_{\text {(probe) }}=-2.24+19.67(\mathrm{~s}) \mathrm{r}=0.9542, \mathrm{sd}=0.8537$

It is possible that the lower significance of $\beta_{\mathrm{s}}$ is due to inefficient interactions between the solvent (as electron donor) and the heterocyclic quaternary nitrogen of the zwitterionic probe. For example, whereas the $\left(\mathrm{CH}_{3}\right)_{4} \mathrm{~N}^{+}$ion has no effect on the structure of water, $\left(\mathrm{n}-\mathrm{C}_{4} \mathrm{H}_{9}\right)_{4} \mathrm{~N}^{+}$has a net structure-enhancing effect, due to hydrophobic hydration of the alkyl groups. ${ }^{22}$ On the other hand, addition of $\beta_{\mathrm{S}}$ to the equation that describes the dependence of Gibbs free energies of solution of tetra-alkylammonium halides on solvent properties did not increase the overall correlation coefficient, so that $\beta_{\mathrm{s}}$ was dropped. ${ }^{23}$

Table 2. Standardized regression coefficients of Equation 3 for some of the probes shown in Figure 2a

\begin{tabular}{lcccccc}
\hline probe & $\beta_{\text {statistical }}$ (a) & $\beta_{\text {statistical }}$ (b) & $\beta_{\text {statistical }}$ (s) & $\beta_{\text {statistical }}$ (p) & $\mathrm{r}^{2}$ & $\mathrm{n}^{\mathbf{b}}$ \\
\hline $\mathrm{MePMBr}_{2}$ & 0.81 & 0.02 & 0.46 & -0.24 & 0.9258 & 36 \\
$\mathrm{BuPMBr}_{2}$ & 0.80 & -0.01 & 0.44 & -0.21 & 0.9334 & 35 \\
$\mathrm{OcPMBr}_{2}$ & 0.79 & -0.01 & 0.43 & -0.22 & 0.9317 & 35 \\
$\mathrm{RB}$ & 0.80 & 0.03 & 0.47 & -0.06 & 0.8677 & 57 \\
$\mathrm{WB}$ & 0.80 & -0.02 & 0.37 & -0.08 & 0.9412 & 25 \\
$\mathrm{QB}$ & 0.85 & 0.01 & 0.29 & -0.02 & 0.9415 & 24 \\
\hline
\end{tabular}

a- Data are taken from ref. 17. The scales of $\alpha_{\mathrm{s}}, \beta_{\mathrm{s}}$, etc. are different; use of the standardized values, $\beta_{\text {statistical }}$, makes the regression coefficients of Equation 3 directly comparable; $b$ - number of solvents tested.

As shown in Table 1, solvatochromic parameters can be used to explain the effects of solvents on chemical reactions. In fact, solvatochromism of these dyes seems to be a good model for several reactions, as shown in Figure 4 for the $\mathrm{pH}$-independent hydrolysis of two esters, 4-nitrophenyl chloroformate (NPCF) and 4-nitrophenyl heptafluorobutyrate (NPFB), in acetonitrile-water mixtures, over a wide water concentration range. ${ }^{24}$ The resemblance between the two plots, i.e., reduced $\mathrm{k}_{\mathrm{obs}}$ versus $\log$ (water) and reduced $\mathrm{E}_{\mathrm{T}}$ (probe) versus log (water) is remarkable because the phenomena involved are quite distinct; acyl transfer reactions, and intra-molecular charge transfers, respectively. Note also that the responses to the composition of the binary solvent mixture of the less hydrophobic ester, NPCP, and the less hydrophobic probe, QB, are similar; the same applies to the more hydrophobic ester, NPFB, and probe, WB. This resemblance has also been noted for other acyl-transfer reactions; ${ }^{25}$ it can be taken to mean that the phenomenon involved in solvatochromism serves as model for reactions where there is a large difference in dipolarity between the reactants and the corresponding activated complexes.

The use of solvatochromic parameters in order to explain the effects of solvents appears to be of wide applicability. Thus we have studied cellulose swelling by twenty protic and sixteen aprotic solvents, and correlated the extent of swelling with several solvent properties, including solvatochromic parameters, Gutmann's solvent acceptor and donor numbers, and Hildebrand's solubility parameters. Swelling of cellulose is an important initial step in several processes including, inter alia, obtaining regenerated fibers (e.g., Rayon and Lyocel); mercerization, and functionalization of cellulose under heterogeneous and homogeneous reaction conditions. ${ }^{26}$ Our results have shown that the use of solvatochormic parameters in order to describe the dependence of cellulose swelling on solvent properties resulted in better correlations coefficients, as compared with those based on Gutmann's, or Hildebrand's solubility parameters. ${ }^{27}$ In fact, we were able to correlate the swelling of twenty eight protic and aprotic solvents simultaneously with the solvent molar volume, $\beta_{\mathrm{S}}$, and $\pi_{\mathrm{s}}^{*}\left(\alpha_{\mathrm{S}}\right.$ was excluded because the dipolar aprotic solvents carry no acidic hydrogens). ${ }^{27}$ This is remarkable because previous 

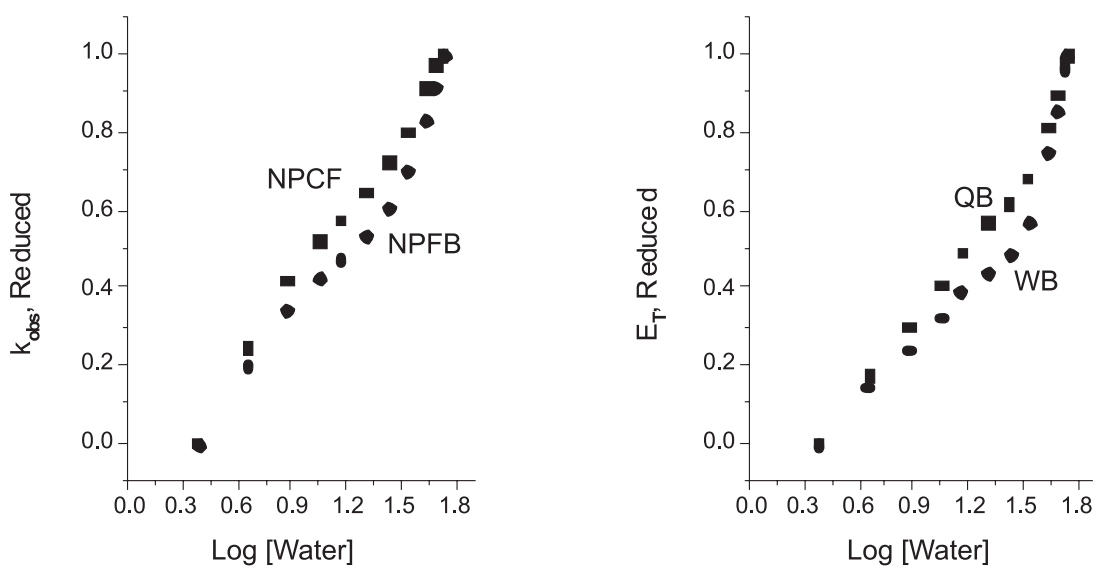

Figure 4. Plots of the dependence of $\log \left(k_{\text {obs }}\right)$ and of $E_{T}$ (probe) on $\log \left[\right.$ water], at $25^{\circ} \mathrm{C}$. Reduced $\log \left(k_{\text {obs }}\right)$ and reduced $E_{T}$ (probe) are employed, so that results of different species (esters, probes) may be directly compared. The esters are 4-nitrophenyl chloroformate (NPCF) and 4-nitrophenyl heptafluorobutyrate (NPFB). Reproduced from ref. 24 with permission of Wiley-Blackwell

work on swelling has either used a very limited number of solvents, or employed solvents of the same nature, either protic or aprotic. ${ }^{28}$ This success indicates that the same solute-solvent interactions mechanisms are relevant to probe solvation and cellulose swelling.

Question (iii) above is concerned with solvation by solvent mixtures. I concentrate here on binary mixtures where hydrogen bonding between its components is operative, e.g., those of water $(\mathrm{W})$ and a miscible molecular solvent, or an ionic liquid (S). Ternary solvent mixtures can be treated, in principle, by using a similar approach. ${ }^{29}$ Solvation by these media is complex because of a simple fact: The composition of the solvation shell of solvatochromic probes, and presumably other species of interest is seldom, if ever, equal to the composition of the bulk binary mixture. There is "preferential solvation" by one component of the medium. One possible reason for this preferential solvation is the so-called "dielectric enrichment". This term denotes enrichment of the probe solvation shell in the solvent of higher $\varepsilon_{\mathrm{r}}$, due to probe dipole-solvent dipole interactions. This interaction, if it occurs, implies a positive deviation in the $\mathrm{E}_{\mathrm{T}}$ (probe) versus $\chi_{\text {higher }\left(\varepsilon_{\mathrm{r}}\right)}$ plot ( $\chi$ is concentration on the mole fraction scale), even when the Onsager dielectric function of the mixture is a linear function in $\chi_{\text {higher }\left(\varepsilon_{\mathrm{r}}\right)}{ }^{4}$ Indeed, $\mathrm{E}_{\mathrm{T}}$ (probe) were found to be non-linear functions of $\chi_{\text {higher }\left(\varepsilon_{\mathrm{r}}\right)}$ for the ideal binary mixtures cyclohexane-THF and cyclohexane-1-butanol. ${ }^{30}$ This interaction mechanism is nonspecific and is, therefore, independent of probe-structure. A more fundamental reason for the non ideal solvation behavior is, however, solvent micro-heterogeneity, i.e., where one component of the mixed solvent prefers a molecule of the same type. A large body of experimental data and theoretical calculations, e.g., of the Kirkwood-Buff integral functions (that describe W-W, S-S, and S-W interactions), has shown that many binary mixtures are micro-heterogeneous; there exist micro-domains composed of organic solvent surrounded by water, and of water solvated by organic solvent. The onset and composition of these micro-domains depend on the pair of solvents. There exists the possibility of solvation of the probe by one of the two solvent micro-domains. ${ }^{4,610,11}$ Examples of non-linear solvation behavior at different temperatures are shown in Figures 5 and 6, for binary mixtures of $\mathrm{W}$ with methanol, $\mathrm{MeOH},{ }^{14}$ and 1-allyl-3-methyl-; 3-(1-butyl), and 3(1-hexyl)imidazolium chloride ionic liquids, ILs, $\mathrm{AlMeImCl}, \mathrm{AlBuImCl}$, and $\mathrm{AlHeImCl}$, respectively. ${ }^{15}$

We have introduced a model in order to describe solvation in these media, based on the principle that the medium should be considered as composed of the two precursor solvents plus a "complex" one, formed by the hydrogen-bond species (W.....S). The solvent exchange equilibria involved are:

$$
\begin{aligned}
\mathrm{S}+\mathrm{W} & \rightleftharpoons \mathrm{S}-\mathrm{W} \\
\operatorname{Probe}(\mathrm{S})_{\mathrm{m}}+\mathrm{m}(\mathrm{S}-\mathrm{W}) & \rightleftharpoons \operatorname{Probe}(\mathrm{S}-\mathrm{W})_{\mathrm{m}}+\mathrm{m} \mathrm{S} \\
\operatorname{Probe}(\mathrm{W})_{\mathrm{m}}+\mathrm{m}(\mathrm{S}-\mathrm{W}) & \rightleftharpoons \operatorname{Probe}(\mathrm{S}-\mathrm{W})_{\mathrm{m}}+\mathrm{m} \mathrm{W}
\end{aligned}
$$

These, after algebraic manipulation, lead to the following solvent exchange equilibria, termed solvent "fractionation factors" $\varphi::^{10,11}$

$$
\begin{aligned}
\varphi_{\mathrm{W} / \mathrm{S}} & =\frac{x_{\mathrm{W}}^{\text {Probe }} / x_{\mathrm{S}}^{\text {Probe }}}{\left(x_{\mathrm{W}}^{\mathrm{Bk} \text { Effective }} / x_{\mathrm{S}}^{\mathrm{Bk} ; \text { Effective }}\right)^{\mathrm{m}}} \\
\varphi_{\mathrm{S}-\mathrm{W} / \mathrm{S}} & =\frac{x_{\mathrm{S}-\mathrm{W}}^{\text {Probe }} / x_{\mathrm{S}}^{\text {Probe }}}{\left(x_{\mathrm{S}-\mathrm{W}}^{\mathrm{Bk} \text { Effective }} / x_{\mathrm{S}}^{\mathrm{Bk} ; \text { Effective }}\right)^{\mathrm{m}}}
\end{aligned}
$$
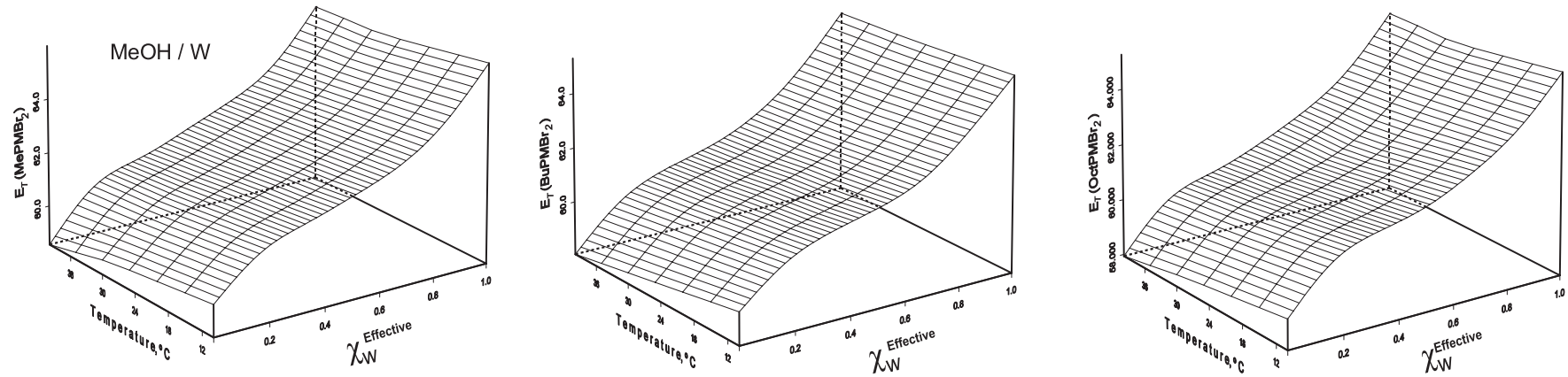

Figure 5. Solvent polarity/temperature/solvent composition contours for $\mathrm{MePMBr}_{2}, \mathrm{BuPMBr}_{2}$ and $\mathrm{OcPMBr}_{2}$, in $\mathrm{MeOH} / \mathrm{W}$. Reproduced from ref. 14 with permission of ACS 
AlMelmCl

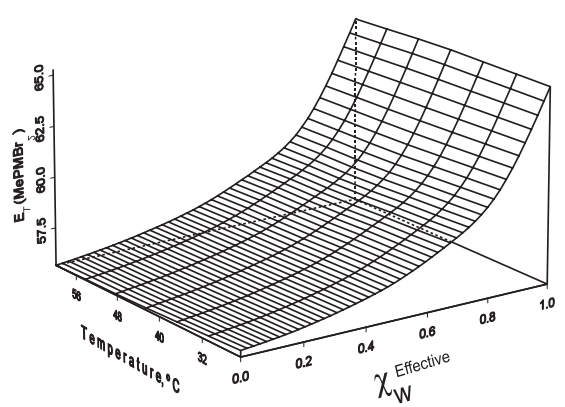

AlBulmCl

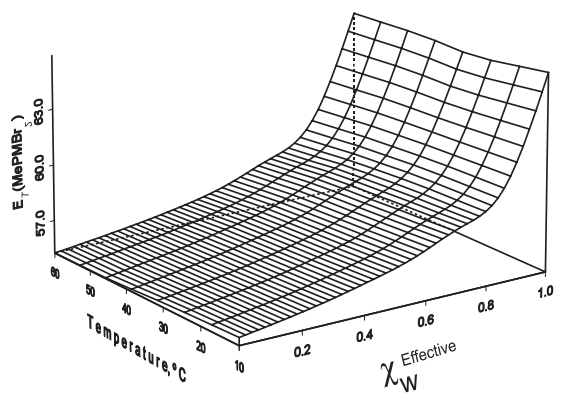

AlHxImCl

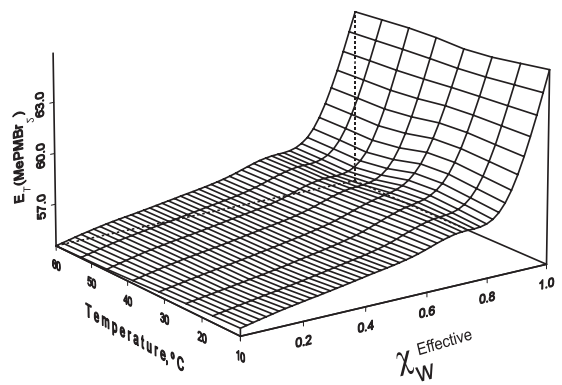

Figure 6. Solvent polarity-temperature-solvent composition contours for $\mathrm{MePMBr}_{2}$ in mixtures of water with AlMeImCl, AlBuImCl, and AlHxImCl, respectively. Reproduced from ref. 15 with permission of RSC

$$
\varphi_{\mathrm{S}-\mathrm{W} / \mathrm{W}}=\frac{x_{\mathrm{S}-\mathrm{W}}^{\text {Probe }} / x_{\mathrm{W}}^{\text {Probe }}}{\left(x_{\mathrm{S}-\mathrm{W}}^{\mathrm{Bk} \text { Effective }} / x_{\mathrm{W}}^{\mathrm{Bk} ; \text { Effective }}\right)^{\mathrm{m}}}
$$

where $(\mathrm{m})$ represents the number of solvent molecules whose exchange in the probe solvation shell affects $\mathrm{E}_{\mathrm{T}}$ (probe); usually $\mathrm{m} \leq 2$, Bk refers to bulk solvent, and the concentrations of the solvent species are "effective" not analytical ones. Note that (m) should not be confused with the total number of solvent molecules that solvate the probe. This model enabled us to calculate the effective compositions in the solvation shells of these probes for a large number of mixtures of water with molecular solvents and ILs. Examples of the results of these calculations for the above-mentioned ILs are shown in Figure $7 .{ }^{15}$

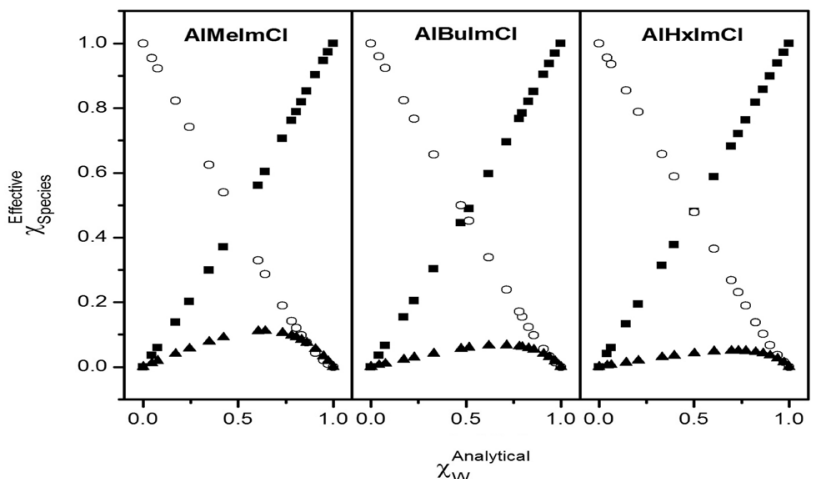

Figure 7. Representative dependence of the concentrations of the species in the $I L-W$ binary mixtures on the length of $R$ of AlRImCl. The data shown are for $25^{\circ} \mathrm{C}$; the symbols employed are $\mathrm{O}, \mathbf{\square}, \mathbf{\Delta}$ for $I L, W$, and the $I L-W$ 1:1 complex, respectively. Reproduced from ref. 15 with permission of RSC

The information about preferential solvation is extracted from the values of the different $\varphi$ of Equation 8-10. For example, for $\varphi_{\mathrm{w} / \mathrm{s}}>1$, the solvation shell is richer in (W) than the bulk mixture; the converse holds for $\varphi_{\mathrm{w} / \mathrm{S}}<1$, i.e., the probe is preferentially solvated by (S). Finally, a solvent fractionation factor of unity indicates an ideal behavior, i.e., solvation shell and bulk mixture have equal compositions. The same line of reasoning applies to $\varphi_{\text {s-w/S }}$ (complex solvent substituting $S$ ) and $\varphi_{\text {S-W/W }}$ (complex solvent substituting $W$ ), Equations 9 and 10, respectively.

Examples of this type of analysis for W-S are shown in Table 3 for mixtures of water with alcohols and the above-mentioned ILs, respectively. As shown, the values of $(\mathrm{m})$ indicate that a small number of solvent molecules (1 to 2) affect the energy of intra-molecular CT.
For all binary mixtures, the solvation is preferential by $\mathrm{S}$ and, much more efficiently by W-S.

Table 3. Values of $\varphi$ for solvent exchange for alcohols and ionic liquids, at $25^{\circ} \mathrm{C}^{14,15}$

\begin{tabular}{lcccc}
\hline Solvent & $m$ & $\varphi_{\mathrm{w} / \mathrm{s}}$ & $\varphi_{\mathrm{s}-\mathrm{w} / \mathrm{s}}$ & $\varphi_{\mathrm{s}-\mathrm{w} / \mathrm{w}}$ \\
\hline Methanol & 1.352 & 0.466 & 3.212 & 6.892 \\
Ethanol & 1.356 & 0.394 & 13.868 & 35.153 \\
1-Propanol & 1.695 & 0.263 & 184.41 & 701.21 \\
2-Propanol & 1.331 & 0.267 & 30.540 & 114.46 \\
AlMeImCl & 1.183 & 0.078 & 1.285 & 16.474 \\
AlBuImCl & 0.973 & 0.075 & 1.428 & 19.040 \\
AlHeImCl & 0.858 & 0.070 & 1.468 & 20.954 \\
\hline
\end{tabular}

I finish this account on solvation by a brief discussion of thermosolvatochromism, i.e., effect of increasing temperature on solvation, see Figures 5 and 6. We have studied solvatochromism in the temperature range, where feasible, from 10 to $60^{\circ} \mathrm{C}$. In all cases, the values of $\mathrm{E}_{\mathrm{T}}$ (probe) were found to decrease as a function of increasing the temperature. Raising the latter decreases the structure of the pure solvent, and the hydrogen-bonding between its components, affecting solvation. This change makes it possible to calculate the energy of desolvation of the probe. Although the desolvation of reactants and activated complexes is known to contribute to temperature effects on reaction rates, there is no obvious way to calculate its contribution to $\Delta \mathrm{H}^{\neq}$from the Arrhenius plot. This desolvation energy is readily calculated from thermo-solvatochromism. Our results have shown that the magnitude of this energy is sizeable (e.g., range from 2.1 to 3.7 $\mathrm{kcal} \mathrm{mol}{ }^{-1}$ over a $50{ }^{\circ} \mathrm{C}$ range for WB in aqueous alcohols) relative to the activation enthalpies of many organic reactions. ${ }^{10,11}$

\section{CONCLUSIONS}

A simplified, non-mathematical description of solvation is provided. Effects of solvents and solvent mixtures on distinct chemical phenomena can be rationalized by a common reasoning, namely, analysis of the solvent effects in terms of a linear combination of properties such as solvent acidity, basicity, dipolarity/polarizability, and lipophilicity. Evaluation of the relative importance of these interactions requires studying solvatochromism of probes of adequate molecular structures. Solvatochromism in binary solvent mixtures can be described by a general mechanism, based on the presence in solution of hydrogen bonded species between the two 
precursor solvents. All probes studied are preferentially solvated by the organic solvent, or IL and, much more efficiently, by the (S-W) hydrogen-bonded species. Values of $\mathrm{E}_{\mathbf{T}}$ (probe) decrease as a function of increasing the temperature because of effects of the latter on the structures of water and the organic component, and on their mutual interactions. The dependence of $\mathrm{E}_{\mathrm{T}}$ (probe) on the composition of the binary mixture, the properties of the probe, and the temperature can be fruitfully employed in order to better explain reactivity data, e.g., the (complex) dependence on medium composition of rate constants and activation parameters. Studies of solvatochromism and thermosolvatochromism are becoming increasingly important because of the current interest in the use of green solvents, e.g., super-critical $\mathrm{CO}_{2},{ }^{30}$, and ILs. ${ }^{30}$

\section{ACKNOWLEDGEMENTS}

I would like to thank FAPESP (State of São Paulo Research Foundation) for financial support; $\mathrm{CNPq}$ (National Council for Scientific and Technological Research) for a research productivity fellowship; my research co-workers L. P. Novaki, E. B. Tada, P. L. Silva, M. Antonious, C. T. Martins, M. S. Lima, L. C. Fidale, E. L. Bastos, B. M. Sato, and P. A. R. Pires for doing the work reported here.

\section{REFERENCES}

1. Kemp, D. S. ; Paul, K. G.; J. Am. Chem. Soc. 1975, 97, 7305; Grate, J. W.; Mcgill, R. A.; Hilvert, D.; J. Am. Chem. Soc. 1993, 115, 8577.

2. Pytela, O.; Coll. Czech. Chem. Commun. 1988, 53, 1333.

3. Politzer, P.; Murray, J. S.; Quantitative Treatments of Solute-Solvents Interactions, Elsevier: New York, 1994, p. 230.

4. Suppan, P.; Ghoneim, N.; Solvatochromism, The Royal Society of Chemistry: Cambridge, 1997, p. 21.

5. Abboud, J.-L. M.; Notario, R.; Pure Appl. Chem. 1999, 71, 645.

6. Hefter, G.; Marcus, Y.; Waghorne, W. E.; Chem. Rev. 2002, 102, 2773.

7. Reichardt, C.; Solvents and Solvent Effects in Organic Chemistry, $3^{\text {rd }}$ edn., VCH: Weinheim, 2003, p. 5, 329, 389.

8. Reichardt, C.; Pure Appl. Chem. 2004, 76, 1903.

9. Reichardt, C.; Green Chem. 2005, 7, 339.

10. El Seoud, O. A.; Pure Appl. Chem. 2007, 79, 1135.

11. El Seoud, O. A.; Pure Appl. Chem. 2009, 81, 697.

12. Kamlet, M. J.; Taft, R. W.; Prog. Phys. Org. Chem. 1981, 13, 485;
Abraham, M. H.; Grellier, P. L.; Abboud, J.-L. M.; Doherty, R. M.; Taft, R. W.; Can. J. Chem. 1988, 66, 2673; Laurence, C.; Nicolet, P.; Dalati, M. T.; Abboud, J.-L. M.; Notario, R.; J. Phys. Chem. 1994, 98, 5807.

13. Catálan, J.; J. Phys. Chem. B 2009, 113, 5951.

14. Martins, C. T.; Lima, M. S.; El Seoud, O. A.; J. Org. Chem. 2006, 71, 9068.

15. Sato, B. M.; de Oliveira, C. G.; Martins, C. T.; El Seoud, O. A.; Phys. Chem. Chem. Phys. 2010, 12, 1764.

16. Coleman, C. A.; Murray, C. J.; J. Org. Chem. 1992, 57, 3578.

17. Silva, P. L.; Pires, P. A. R.; Trassi, M. A. ; El Seoud, O. A.; J. Phys. Chem. B 2008, 112, 14976.

18. Chandramani, R.; Devaraj, N.; J. Chem. Soc., Faraday Trans. 21980 , 76,1055 .

19. Tada, E. B.; Novaki, L. P.; El Seoud, O. A.; Langmuir 2001, 17, 652; Tada, E. B.; El Seoud, O. A.; Prog. Colloid Polym. Sci. 2002, 121, 101.

20. Lagalante, A. F.; Spadi, M.; Bruno, T. J.; J. Chem. Eng. Data 2000, 45, 382.

21. Dawber, J. D.; Ward, J.; Williams, R. A.; J. Chem. Soc., Faraday Trans. 1 1988, 84, 713.

22. El Seoud, O. A.; J. Mol. Liq. 1997, 72, 85.

23. Taft, R. W.; Abraham, M. H.; Doherty, R. M.; Kamlet, M. J.; J. Am. Chem. Soc. 1985, 107, 3105.

24. El Seoud, O. A.; Siviero, F.; J. Phys. Org. Chem. 2006, 19, 793.

25. El Seoud, O. A.; El Seoud, M. I.; Farah, J. P. S.; J. Org. Chem. 1997, 62, 5928.

26. Krässig, H. A.; Cellulose structure, accessibility and reactivity, Gordon and Breach Science: New York, 1993, p. 167.

27. El Seoud, O. A.; Fidale, L. C.; Ruiz, N.; D’Almeida, M. L. ; Frollini, E; Cellulose 2008, 15, 371; Fidale, L. C.; Ruiz, N.; Heinze, T.; El Seoud, O. A.; Macromol. Chem. Phys. 2008, 209, 1240.

28. Boluk, Y.; Cellulose 2005, 12, 577; Mantanis, G. I.; Young, R. A.; Rowell, R. M.; Holzforschung 1994, 48, 480; Mantanis, G. I.; Young, R. A.; Rowell, R. M.; Cellulose 1995, 2, 1.

29. Maitra, A.; Bagchi, S.; J. Phys. Chem. B 2008, 112, 2056; Maitra, A.; Bagchi, S.; J. Phys. Chem. B 2008, 112, 9847.

30. Martins, C. T.; Lima, M. S.; El Seoud, O. A.; J. Phys. Org. Chem. 2005, $18,1072$.

31. Leitner, W.; Acc. Chem. Res. 2002, 35, 746; Dupont, J.; de Souza, R. F.; Suarez, P. A. Z.; Chem. Rev. 2002, 102, 3667; El Seoud, O. A.; Koschella, A.; Fidale, L. C.; Dorn, S.; Heinze, T.; Biomacromolecules 2007, 8, 2629. 